\title{
RADIATIVE AVALANCHE DRIVEN BY A CIRCUMNUCLEAR STARBURST TORUS
}

\author{
KEN OHSUGA \\ Institute of Physics, University of Tsukuba \\ Tsukuba, Ibaraki 305, Japan; ohsuga@het.ph.tsukuba.ac.jp \\ AND \\ MASAYUKI UMEMURA \\ Center for Computational Physics, University of Tsukuba \\ Tsukuba, Ibaraki 305, Japan; umemura@rccp.tsukuba.ac.jp
}

Recently, a novel mechanism for fueling active galactic nuclei (AGNs) has been proposed by Umemura et al. $(1997 \mathrm{a}, \mathrm{b})$. That is a radiative avalanche, in which a rotating gas disk sheds angular momentum due to the radiation drag force exerted by starlight from circumnuclear starbursts, so that the mass accretion onto nuclei is driven. Originally, a thin ring of a starburst region has been assumed for simplicity. However, recent observations have revealed that circumnuclear starburst rings have radial extension of $\sim 10 \mathrm{pc}$ up to kpc, and they often consist of compact star clusters of $<10 \mathrm{pc}$ Thus, the ratio of the half thickness to curvature radius of the ring can be minimally less than one tenth when all the star clusters are aligned on a plane of an inner gas disk, while the ratio could be larger if the distributions of star clusters are extended due to some velocity dispersions.

Here, we explore the effects of extended starburst regions. For this purpose, assuming a geometry of torus, the radiation force is numerically calculated including the effects of optical depth with taking account of viewing angles from starburst regions. For the present, we do not take the radial radiation force into consideration.

As a result, we found the present results and the previous results for a thin ring of starburst (Umemura et al. 1997b) are in good agreement in a fairly optically thin disk (the total face-on optical depth, $\tau_{0},<0.1$ ), while the present ones is far smaller than the previous ones in $\tau_{0}>0.1$. In Figure 1, the mass accretion rate is shown for an optical thick disk and Keplerian rotation. Here, two cases of half thickness of a starburst torus 


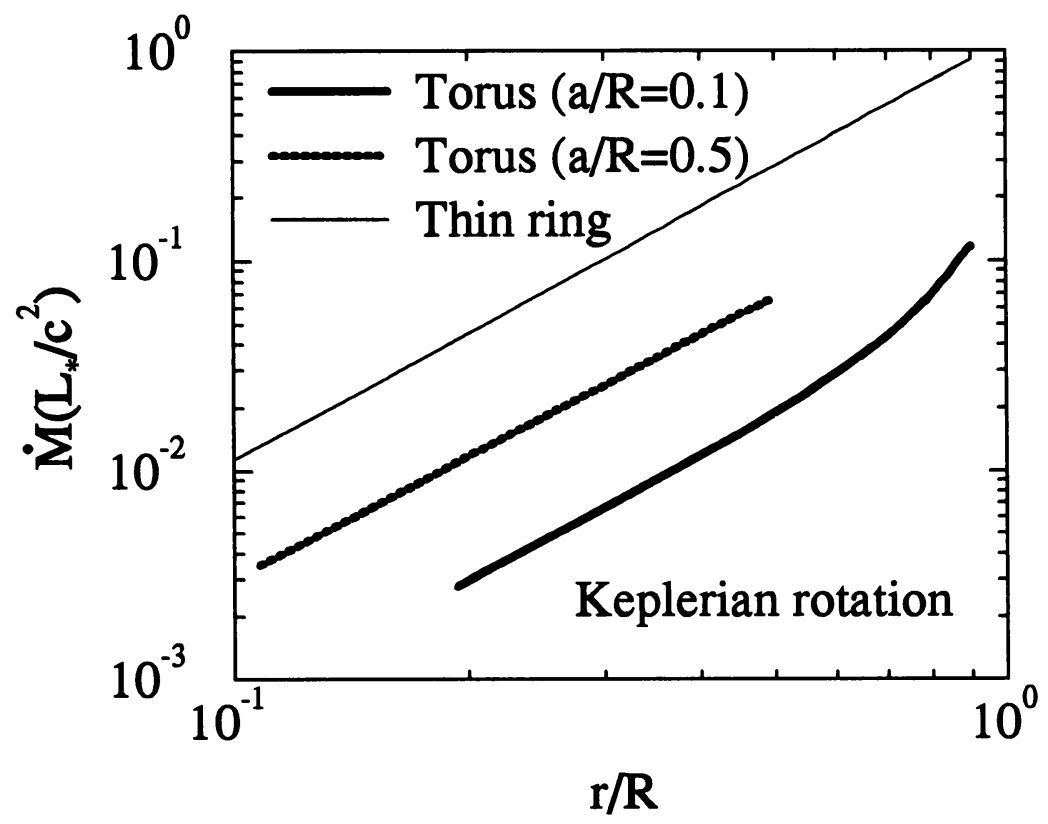

Figure 1. The mass accretion rate by a radiative avalanche is shown in units of $L_{*} / c^{2}$, where $L_{*}$ is the bolometric luminosity of a starburst torus, as a function of radius, $r$, normalized by the curvature radius of torus, $R$, for $a / R=0.1$ (solid curve) and 0.5 (dotted curve), with half thickness of the torus, $a$. Here, an optically thick disk and Keplerian rotation is assumed. A thin dashed line is the previous estimation by Umemura et al.

are considered, i.e., $a / R=0.1$ and $a / R=0.5$. So, the previous results for a thin ring of starburst (Umemura et al. 1997b) is also shown for comparison in Figure 1. As seen in this figure, the mass accretion rate has a common tendency that it increases with increasing radii. Therefore, we find the viewing-angle effects lead to the reduction by a factor of $\sim a / R$ throughout the disk. Incidentally, although not shown in Figure 1, the rate for a rigid rotation disk is found to be negative, e.g., $\dot{M} \sim-0.02 L_{*} / c^{2}$, also, for a flat rotation disk, to be $\sim 0.02 L_{*} / c^{2}$ in the vicinity of the torus for $a / R=0.1$.

In this work, we have not taken the radial radiation force into consideration. It is important to couple an azimuthal component of the moment equation with a radial component, for the purpose of investigating the gas motion or mass accretion rate more exactly (Umemura et al. 1997b). Such analysis will be done in a further work.

\section{References}

Umemura M., Fukue J., Mineshige S. (1997a) Radiative Avalanche: Starburst Induced Fuelling to AGNs, Astrophysical Journal Letters, Vol.479 no.2, pp. 97-100

Umemura M., Fukue J., Mineshige S. (1997b) Radiative Avalanche onto Galactic Nuclei Induced by Circumnuclear Starbursts, submitted to Astrophysical Journal 